\title{
Evaluating initial spine trauma response: injury time to trauma center in PA, USA.
}

James Harrop, MD

Thomas Jefferson University

George M. Ghobrial, MD

Thomas Jefferson University

Rohan Chitale, MD

Thomas Jefferson University

Kelly Krespan

Thomas Jefferson University

=al4tra Afforizżiadditional works at: https://jdc.jefferson.edu/neurosurgeryfp Thomas Jefferson University

Part of the Orthopedics Commons

\section{$\frac{\text { Let us know how access to this document benefits you }}{\text { See next page for additional authors }}$}

\section{Recommended Citation}

Harrop, MD, James; Ghobrial, MD, George M.; Chitale, MD, Rohan; Krespan, Kelly; Odorizzi, Laura; Fried, Tristan; Maltenfort, PhD, Mitchell; Cohen, MD, Murray; and Vaccaro, MD, Alex R., "Evaluating initial spine trauma response: injury time to trauma center in PA, USA." (2014).

Department of Neurosurgery Faculty Papers. Paper 67.

https://jdc.jefferson.edu/neurosurgeryfp/67

This Article is brought to you for free and open access by the Jefferson Digital Commons. The Jefferson Digital Commons is a service of Thomas Jefferson University's Center for Teaching and Learning (CTL). The Commons is a showcase for Jefferson books and journals, peer-reviewed scholarly publications, unique historical collections from the University archives, and teaching tools. The Jefferson Digital Commons allows researchers and interested readers anywhere in the world to learn about and keep up to date with Jefferson scholarship. This article has been accepted for inclusion in Department of Neurosurgery Faculty Papers by an authorized administrator of the Jefferson Digital Commons. For more information, please contact: JeffersonDigitalCommons@jefferson.edu. 


\section{Authors}

James Harrop, MD; George M. Ghobrial, MD; Rohan Chitale, MD; Kelly Krespan; Laura Odorizzi; Tristan Fried; Mitchell Maltenfort, PhD; Murray Cohen, MD; and Alex R. Vaccaro, MD 
SCI Time to Treatment 1

\title{
As submitted to:
}

\author{
Journal of clinical neuroscience : official journal of the Neurosurgical Society \\ of Australasia.
}

And later published as:

\section{Evaluating Initial Spine Trauma Response: Injury Time to Trauma Center in PA, USA.}

\author{
Volume 21, Issue 10, pp. 1725-9
}

\section{October 2014}

\section{DOI: 10.1016/j.jocn.2014.03.011}

James S. Harrop, MD (james.harrop@jefferson.edu)

George M. Ghobrial MD (George.ghobrial@jeffersonhospital.org)

Rohan Chitale MD (rohan.chitale@jeffersonhospital.org)

Kelly Krespan (kelly.krespan@jefferson.edu)

Laura Odorizzi (laura.odorizzi@jefferson.edu)

Tristan Fried (tristanbfried@gmail.com)

Mitchell Maltenfort, PhD (mitchell.maltenfort@ rothmaninstitute.com)

Murray Cohen, MD (murray.cohen@jefferson.edu)

Alexander Vaccaro, MD (alexander.vaccaro@rothmaninstitute.com)

Running Title: Spine Trauma Response

Departments of Neurological and Orthopedic Surgery, Thomas Jefferson University Hospital, 909 Walnut Street, Philadelphia, PA 19107

Conflicts of Interest and Sources of Funding: Dr. Alex Vaccaro has Stock/ Stock Option Ownership Interests in Spine Medica; Computational Biodynamics; Progressive Spinal Technologies; Spinology; Small Bone Innovations; NeuCore; Cross Current; Syndicom; In Vivo; Flagship Surgical; Advanced Spinal Intellectual Properties; Cytonics; Bonovo Orthopaedics; Electrocore; Gamma Spine; Location Based Intelligence; FlowPharma; R.S.I.; Replication Medica; Globus; K-2 Medical; Paradigm Spine; Stout Medical; Innovative Surgical Design; Spinicity; Rothman Institute and Related Properties. Dr Vaccaro also has receives royalty payments from DePuy; Medtronics; Stryker Spine; Biomet Spine; Globus; Aesculap; Nuvasive. He is involved with consulting/independent contracting for Gerson Lehrman Group; Guidepoint Global; Medacorp; Stout Medical; Innovative Surgical Design. He serves on scientific advisory board/board of directors/committees including AO Spine; Innovative Surgical Design; Association of Collaborative Spine Research; Spinicity. He has Institutional/ Educational Grant from Stryker Spine; Nuvasive; Cerapedics.

For the remaining authors, no conflicts are declared.

There are no sources of funding to report. 
This manuscript does not appear in any other publication, in full or in part, except as an abstract for presentation at the Congress of Neurological Surgeons conference, October 19-23, 2013, in San Francisco, CA.

Acknowledgements: None

\section{Corresponding Author:}

James S. Harrop, M.D.

Department of Neurological Surgery

Thomas Jefferson University Hospital

909 Walnut Street

Philadelphia, PA 19107

james.harrop@jefferson.edu

Tel: 215-955-7959

Fax: 215-503-9170

Keywords: SCI, timing, trauma, Cranial, Neurologic, transportation 
SCI Time to Treatment 3

\begin{abstract}
Historical perceptions regarding the acuity of traumatic spinal cord injury has led to considerable disparity in triage to tertiary care centers. This article retrospectively reviews a large regional trauma database to analyze whether the diagnosis of spinal trauma affected patient transfer timing and patterns. The Pennsylvania Trauma database, was retrospectively reviewed. All acute trauma patients' entries for level I and II centers were categorized diagnosis, mechanism, and location of injury, analyzing transportation modality and its influence on time of arrival. 1162 trauma patients were identified, (1014 blunt injuries, 135 penetrating injuries and 12 other) with a mean transport time of 3.9 hours and a majority of patients arriving within seven hours $(>75 \%)$. Spine trauma patients had the longest mean arrival time ( 5.2 hours) compared to blunt trauma (4.2 hours), cranial neurologic injuries (4.35 hours), and penetrating injuries $(2.13$ hours, $\mathrm{p}<0.0001)$. There was a statistically significant correlation between earlier arrivals and both cranial trauma $(\mathrm{p}=0.0085)$ and penetrating trauma $(\mathrm{p}<0.0001)$. The fastest modality was a firerescue $(0.93 \mathrm{hrs})$ or police $(0.63 \mathrm{hrs})$ vehicle with Philadelphia County $(1.1 \mathrm{hr})$ having the quickest arrival times. Most trauma patients arrived to a specialty center within 7 hours of injury. However subsets analysis revealed that spine trauma patients had the greatest transit times. Present research trials for spinal cord injuries suggest earlier intervention may lead to improved recovery. Therefore, it is important to focus on improvement of the transportation triage system for traumatic spinal patients.
\end{abstract}

Keywords: SCI, timing, trauma, Cranial, Neurologic, transportation, epidemiology, triage, regional trauma database, cervical spine injury 


\section{SCI Time to Treatment 4}

\section{Introduction}

The development of advanced trauma transportation networks has evolved over the last century. The regionalization and networking of trauma resources and facilities has increased the speed in which severely injured patients can be transported to specialized centers. Concurrently, over the last several decades the treatment of spinal fractures and spinal cord injuries has also significantly improved, with evidence supporting that results may be time sensitive.[1, 2] Spine trauma literature suggests that early decompression and stabilization of spinal trauma patients may improve outcomes and decrease length of stay, morbidities, and, potentially, mortality, particularly, when intervention is within 24 hours. However, the timing, transportation, coordination and mobilization of these spine injured patients are not often directed by the spine surgeons. Unlike penetrating injuries where there is an overall recognition of the need for emergent and rapid transportation, there is a perception that spinal trauma does not require emergent, but only urgent, triage to specialty centers. This article retrospectively reviews a large regional trauma database, The Pennsylvania Trauma Outcome Study (PTOS), to analyze whether the diagnosis of spinal trauma affected patient transfer timing and patterns. Given the relatively increased complexity of this disease, the authors hypothesize that spinal trauma patients have a relatively prolonged transfer time. This would be an important finding since current best evidence guidelines support the urgent decompression and stabilization of unstable traumatic spinal cord injuries.[3-6]

\section{$\underline{\text { Methods }}$}

The Pennsylvania Trauma Systems Foundation’s State Registry, Pennsylvania Trauma Outcome Study (PTOS), is a prospectively collected database that maintains the diagnosis and triage management of the state of Pennsylvania's accredited level I and II trauma centers. This registry is administrated by the Pennsylvania Trauma Systems Foundation (PTSF), a state organization which manages the accreditation process for all Pennsylvania State trauma centers as well as administrates over data submitted directly to the PTOS by the participating centers.[7] The integrity of this submission process is verified by PTSF employees on a biannual basis. A full-time dedicated registry director manages the database, appointed by the board of directors of the PTSF. Specifically, there were 23 adult level I and II trauma centers included in the analysis. (11 level I and 12 level II centers). [figure 1] This trauma registry was retrospectively reviewed over a one year period for the adult (>18yo) trauma admissions from 
1/1/10 and 12/31/10. Patients are distinguished by their trauma center county of origin only, and not by the name of the trauma center.

The patients were categorized per protocol of the PTOS into one of three categories (blunt, penetrating, others/burns). The data was then further categorized into: spine trauma (spine fractures, spinal cord injuries), cranial trauma (i.e. subarachnoid hemorrhage, subdural hematoma, intraventricular hemorrhage, traumatic brain injuries,), modality of transportation (ambulance, fire rescue, helicopter, police, private vehicle, and walk-in), time from injury to arrival at level I-II trauma center, day of the week of injury and county/region of injury. Currently, it is estimated that $88.5 \%$ and $99.3 \%$ of the state's population has access to a level I and level II trauma center within 45 and 60 minutes, respectively.[7]

\section{Statistical Analysis}

A logistic regression was performed, controlling for county or origin, diagnosis, transportation modality, and transportation duration. Specifically, a generalized linear model with quasibinomial family to account for variation beyond that expected in ordinary binomial (logistic) regression was utilized. Significance was set at a probability of 0.05 by convention. Statistical analysis was performed using JMP 7.02 (SAS Institute, Cary NC).

\section{$\underline{\text { Results }}$}

A total of 1161 patients were included in analysis: 1014 blunt injuries, 135 penetrating injuries and 12 patients categorized as other. Mean arrival time to a level I-II facility from onset of injury was 3.95 hours for the entire cohort. The majority of patients arrived within the first seven hours (>75\%). (figure 2) There was no correlation between arrival times and the occurrence of the injury by day of the week (Monday-Sunday) (figure 3).

The investigation of factors influencing trauma patients' arrival times was further carried out through a subanalysis by categorizing patients by initial trauma diagnosis into: (non-craniospinal) penetrating, (non-craniospinal) blunt, spinal and cranial injuries. This analysis revealed that the spinal trauma patients had the longest time from onset of injury to arrival at the level I-II centers, with a mean time of 5.2 hours (figure 4). Patients with blunt trauma (4.2 hours) and cranial neurologic injuries (4.35 hours) had earlier arrival times compared to the spine trauma population. However, the patients with the fastest arrival times from scene to a level I-II center were those with penetrating injuries $(2.13$ hours, $\mathrm{p}<0.0001)$. There was a statistically significant correlation between earlier arrivals and both cranial trauma $(\mathrm{p}=0.0085)$ and penetrating trauma $(\mathrm{p}<0.0001)$. In contradistinction, the spine trauma patients had a significant correlation with longer arrival times $(\mathrm{p}<0.0001)$ 
There was a variety of transportation modalities that were utilized to carry the patients from the injury site to a Level I-II hospital. The fastest modality was a fire-rescue $(0.93 \mathrm{hrs})$ or police $(0.63 \mathrm{hrs})$ vehicle. The next quickest transportation modalities in ascending order were: walk-ins ( $2 \mathrm{hrs}$ ), ambulance (5.2hrs), and private vehicles (5.4hrs). The longest time from onset of injury to arrival at level I-II trauma center was via helicopter transportation (6.98 hrs). (figure 5)

An analysis of transportation time from site of injury to trauma center based on geographic location showed the following results: Philadelphia County (1.1hr), Montgomery County (4.8hrs), Bucks County (5.08hrs), Delaware County (5.6hrs), and Chester County (5.72hrs).(figure 6) In addition, the transportation time in the state of New Jersey was 5.6 hours and "other" was 6.17 hours. Ambulance transportation was the primary modality in all regions: Bucks County 69.4\%, Delaware County 67.1\%, Montgomery County 66.1\%, New Jersey $66.1 \%$, Chester County $62.0 \%$, and other $56.4 \%$. The exception was Philadelphia County where $62.9 \%$ of patients arrived via fire rescue and police vehicles, while ambulances were utilized only $8.5 \%$ of the time.

Philadelphia County has the highest number of trauma centers with seven level I trauma centers.

Therefore, a trauma patient in Philadelphia County has a greater number of transportation opportunities with a shorter distance to travel. This is reflected in the shortest transportation times in this county $(1.1 \mathrm{hrs})$ which was significantly lower than other regions $(\mathrm{p}<0.0001)$.

\section{$\underline{\text { Discussion }}$}

The need for rapid intervention has been previously illustrated in the trauma literature, especially in the case of penetrating trauma for which rapid intervention is required for survival. [8-11] Similarly, neurosurgical trauma patient outcomes, particularly for intracranial injuries, are also related to timing of intervention. $[5,12]$ In USA and Canada, the implementation of trauma centers, paramedic services, and rapid transportation by ambulance and helicopters helped to reduce head injury death rates, deaths at the trauma scene, and deaths upon arrival to the hospital. [11-14] However, room for improvement exists in executing a coordinated transportation and health care system. A study by Lind et al demonstrated that in Australia, deficiencies in timing of intervention for closed head injuries existed.[14] They reviewed 34 closed head injury patients' transportation time to specialty centers over a one year period (2002), finding a mean time of 6.5 hours from injury to arrival.[14] In this study only $33 \%$ of the "local" patients, and none from hospitals outside the city, arrived within 4 hours from the onset of their trauma.[15] These authors noted that there was "no special reasons for delayed transfer," and suggested that a systems-based 
approach among hospitals and changes to triaging protocols could impact these results [15]. This concept of streamlining and organizing transportation systems, networks, and protocols can result in improvement in transportation times and potentially in patient outcomes for defined trauma subsets [16]. Tariq et al[17] demonstrate this potential for improved efficiency through early triage and defining a transportation network in the general trauma population. These authors reviewed 191 penetrating chest injury patients' transportation times at a level II trauma hospital in Pakistan and found a 10.8\% improvement in speed of time from injury to the trauma hospital over a twenty year period, which they attributed to a defined transportation network and triage improvements. [17]

The overall evolution of the rapid transportation system for trauma patients to specialty centers has resulted in improved clinical outcomes and decreased patient's morbidity and mortality.[17, 18] However, there are multiple factors that influence the arrival and transportation of these patients and not all types of trauma patients are treated similarly.[10,19] Although there have been significant advances in the care and treatment of trauma patients in general, there arises the question whether various subsets of traumatic injury patients' management and transportation could be further improved.[20] In the state of Pennsylvania, the present transportation schemes appear to be very successful in transporting patients to level I and II trauma centers in a rapid manner, as evinced by the estimated $99.3 \%$ of the population having access to these centers within 60 minutes, the widely referred to "Golden Hour" of improved outcomes.[7, 10] Furthermore, it was shown by Branas and colleagues[3] that a relatively higher utilization of helicopters are needed to achieve this time window (36.9-42\%), a factor that is more common in rural settings. In contrast, after arrival to a trauma center, the majority of patients arrive to a tertiary care center in less than four hours from the onset of the trauma (figure2). However, transportation times for spine trauma patients are statistically longer than that for other trauma patients irrespective of geographic location.

\section{Rapid intervention in Spinal Cord Injury}

Spinal cord injury has been perceived as an urgent but not emergent clinical disorder partly due to the historical lack of treatment algorithms. However, evidence supports a biological benefit of earlier decompression of traumatic spinal cord injury patients in terms of neurologic recovery as well as a decrease in patients morbidities as illustrated by decreased length of stay and associated hospital costs.[4, 21] Further, recent evidence from the Surgical Treatment for Acute Spinal Cord Injury Study (STASCIS) also demonstrates clinical evidence of a 


\section{SCI Time to Treatment 8}

neurological benefit with early surgery.[1] Recent consensus guidelines from AOSpine and Spine Trauma Study Groups reiterate that early decompression for spinal cord injury (SCI) is considered best practice because it reduces neurologic morbidity/impairment. [1,3] The ability to hasten treatment of spinal cord injured patients will be essential to improve outcomes for these patients.

As witnessed in other trauma populations, wider availability of emergency and trauma systems has decreased death and disability seen in traumatic SCI.[10] Regionalization of these services with a focus on rapid transportation for SCI patients may help to allow SCI patients to be seen, evaluated, and treated promptly by spine specialists. Such evaluation and treatment by spine specialists at SCI centers can result in improved outcomes.[9] Triage of patients to tertiary care centers also improves patient access to investigational therapies. Basic science research has identified secondary injury mechanisms that follow initial spinal cord injury which lead to neuronal cell death and adverse clinical outcomes. Trial medications including riluzole and minocycline attempt to thwart these effects, but these also rely on prompt intervention.[22, 23]

With the goal of prompt intervention in spine trauma in mind, Furlan et al identifies systems-based practices which resulted in transportation delays in SCI patients at the Toronto Western Hospital. They note that the "extrinsic factors" including delays in patient assessment, delays in imaging evaluation, and delays in transportation to SCI centers were modifiable variables that could be changed by revisions in clinical practice guidelines.

Attacking these variables in the Pennsylvania trauma triage system may help to improve the discrepancy seen in transportation times between spine and non-spine trauma.[24] Unfortunately, data on the presence or absence of a neurologic deficit was lacking on the spine trauma database and will need to be improved to derive more meaningful data.

\section{$\underline{\text { Conclusion }}$}

Evaluation of Pennsylvania Trauma Outcome Study (PTOS) illustrates that the majority of trauma patients in the region arrive to a specialty center within 7 hours of injury, with a mean of approximately 4 hours. Among subsets, spine trauma patients suffered the longest transit times. Transportation modalities varied by geographical region as did time from injury site to treatment centers. With growing evidence that treatment speed may affect patient outcome, it is important to focus on triage mechanisms and transportation infrastructure in order to improve the system efficiency in bringing patients to appropriate treatment facilities as soon as possible. 
SCI Time to Treatment 9

\section{$\underline{\text { References }}$}

[1] Fehlings MG, Vaccaro A, Wilson JR, Singh A, D WC, Harrop JS, et al. Early versus delayed decompression for traumatic cervical spinal cord injury: results of the Surgical Timing in Acute Spinal Cord Injury Study (STASCIS). PloS one. 2012;7:e32037.

[2] Schiller MD, Mobbs RJ. The historical evolution of the management of spinal cord injury. Journal of clinical neuroscience : official journal of the Neurosurgical Society of Australasia. 2012;19:1348-53.

[3] Fehlings MG, Wilson JR. Timing of surgical intervention in spinal trauma: what does the evidence indicate? Spine. 2010;35:S159-60.

[4] Furlan JC, Noonan V, Cadotte DW, Fehlings MG. Timing of decompressive surgery of spinal cord after traumatic spinal cord injury: an evidence-based examination of pre-clinical and clinical studies. Journal of neurotrauma. 2011;28:1371-99.

[5] van Middendorp JJ. Letter to the editor regarding: "Early versus delayed decompression for traumatic cervical spinal cord injury: results of the Surgical Timing in Acute Spinal Cord Injury Study (STASCIS)". The spine journal : official journal of the North American Spine Society. 2012;12:540; author reply 1-2.

[6] Cadotte DW, Singh A, Fehlings MG. The timing of surgical decompression for spinal cord injury. F1000 medicine reports. 2010;2:67.

[7] Wilberger J CR, Barbera C, Fontana M, Wargo C, Hoff B, Swade J, Reid S, Reilly P, Weibe D, McWIliams M. White Paper on Needs Assessment For New Trauma Center Development In The Commonwealth of Pennsylvania. Pennsylvania Trauma Systems Foundation; 2014. p. 1-20.

[8] Petri RW, Dyer A, Lumpkin J. The effect of prehospital transport time on the mortality from traumatic injury. Prehospital and disaster medicine. 1995;10:24-9.

[9] Sampalis JS, Denis R, Frechette P, Brown R, Fleiszer D, Mulder D. Direct transport to tertiary trauma centers versus transfer from lower level facilities: impact on mortality and morbidity among patients with major trauma. J Trauma. 1997;43:288-95; discussion 95-6.

[10] Branas CC, MacKenzie EJ, Williams JC, Schwab CW, Teter HM, Flanigan MC, et al. Access to trauma centers in the United States. JAMA : the journal of the American Medical Association. 2005;293:2626-33.

[11] Colohan AR, Alves WM, Gross CR, Torner JC, Mehta VS, Tandon PN, et al. Head injury mortality in two centers with different emergency medical services and intensive care. Journal of neurosurgery. 1989;71:202-7. 
[12] Shackford SR, Mackersie RC, Hoyt DB, Baxt WG, Eastman AB, Hammill FN, et al. Impact of a trauma system on outcome of severely injured patients. Arch Surg. 1987;122:523-7.

[13] Kelly DF, Becker DP. Advances in management of neurosurgical trauma: USA and Canada. World journal of surgery. 2001;25:1179-85.

[14] Klauber MR, Marshall LF, Toole BM, Knowlton SL, Bowers SA. Cause of decline in headinjury mortality rate in San Diego County, California. Journal of neurosurgery. 1985;62:528-31.

[15] Lind CR, Heppner PA, Robins TM, Mee EW. Transfer of intubated patients with traumatic brain injury to Auckland City Hospital. ANZ J Surg. 2005;75:858-62.

[16] Sampalis JS, Denis R, Lavoie A, Frechette P, Boukas S, Nikolis A, et al. Trauma care regionalization: a process-outcome evaluation. J Trauma. 1999;46:565-79; discussion 79-81.

[17] Tariq UM, Faruque A, Ansari H, Ahmad M, Rashid U, Perveen S, et al. Changes in the patterns, presentation and management of penetrating chest trauma patients at a level II trauma centre in southern Pakistan over the last two decades. Interact Cardiovasc Thorac Surg.

2011;12:24-7.

[18] Gervin AS, Fischer RP. The importance of prompt transport of salvage of patients with penetrating heart wounds. J Trauma. 1982;22:443-8.

[19] Aguero-Valverde J, Jovanis PP. Spatial analysis of fatal and injury crashes in Pennsylvania. Accident; analysis and prevention. 2006;38:618-25.

[20] Hartl R, Gerber LM, Iacono L, Ni Q, Lyons K, Ghajar J. Direct transport within an organized state trauma system reduces mortality in patients with severe traumatic brain injury. $\mathrm{J}$ Trauma. 2006;60:1250-6; discussion 6.

[21] Lokkeberg AR, Grimes RM. Assessing the influence of non-treatment variables in a study of outcome from severe head injuries. Journal of neurosurgery. 1984;61:254-62.

[22] Sonmez E, Kabatas S, Ozen O, Karabay G, Turkoglu S, Ogus E, et al. Minocycline treatment inhibits lipid peroxidation, preserves spinal cord ultrastructure, and improves functional outcome after traumatic spinal cord injury in the rat. Spine. 2013;38:1253-9.

[23] Wilson JR, Fehlings MG. Riluzole for Acute Traumatic Spinal Cord Injury: A Promising Neuroprotective Treatment Strategy. World neurosurgery. 2013.

[24] Furlan JC, Tung K, Fehlings MG. Process benchmarking appraisal of surgical decompression of spinal cord following traumatic cervical spinal cord injury: opportunities to reduce delays in surgical management. Journal of neurotrauma. 2013;30:487-91. 


\section{Figure Legend}

Figure 1 Level I and II Trauma centers in Pennsylvania: Participating Trauma Centers in the Pennsylvania trauma registry. 1. Abington Memorial Hospital; 2. Albert Einstein Medical Center; 3. Allegheny General Hospital; 4. Altoona Hospital; 5. Aria Health- Torresdale Campus; 6. The Children's Hospital of Philadelphia. 7. The Children's Hospital of Pittsburgh; 8. Geisinger-Community Medical Center; 9. Conemaugh Memorial Medical Center; 10. Crozer-Chester Medical Center; 11. Geisinger Medical Center; 12. Geisinger Wyoming Valley Medical Center; 13. Hahnemann University Hospital; 14. UPMC Hamot; 15. Lancaster General Hospital; 16. Lehigh Valley Health Network Cedar Crest; 17. Paoli Hospital; 18. Penn State Milton S. Hershey Medical Center; 19. Pocono Medical Center; 20. The Reading Hospital and Medical Center; 21. Robert Packer Hospital; St. Christopher's Hospital for Children. 21. Robert Packer Hospital; 22. St. Christopher's Hospital for Children ; 23. St. Luke's University

Hospital; 24. St. Mary Medical Center; 25. Temple University Hospital; 26. Thomas Jefferson University Hospital;

27. University of Pennsylvania Medical Center; 28. University of Pittsburgh Medical Center (UPMC) Mercy; 29. UPMC Presbyterian; 30. York Hospital;

Figure 2 Trauma Triage Time. A distribution of the duration of patient transfer in hours.

Figure 3. Patient Transfer Volume, by Day. A comparison of patient transfer volumes show a trend for the greatest volume on the weekend, but did not reach statistical significance $(\mathrm{P}<0.005)$.

Figure 4 Transfer Duration by Injury. A comparison of triage duration by injury mechanism, showing prolonged durations of processing and transport in spinal injury patients.

Figure 5 Transfer Duration by Modality of Transportation. A comparison of triage duration by modality of transport showing travel times longest in the helicopter method.

Figure 6. Arrival Time by County. A comparison of patient transfer times by Philadelphia Local Counties. 'Other', specifies additional counties that transfer patients to Philadelphia at a low frequency. 